\title{
THREE-DIMENSIONAL COMPUTER AIDED DESIGN OF A VERTICAL WINNOWER
}

\author{
Yumei Bao*, Saijia Lin, Lijie Weng \\ The MOE Key Laboratory of Mechanical Manufacture and Automation, Zhejiang University \\ of Technology, Hangzhou, Zhejiang Province, P.R.China 310032 \\ * Corresponding author, Address: College of Mechanical \& Electrical Engineering, Zhejiang \\ University of Technology, Hangzhou310032,Zhejiang Province, P. R. China, Tel: +86-571- \\ 88320244, Fax: +86-571-88320837,Email: baoym@zjut.edu.cn
}

\begin{abstract}
The research states home and abroad of the winnowing technology and winnowers are reviewed in brief. For the air duct, the core component of the winnower, the relevant technical parameters in the winnowing process are calculated based on the winnowing principle. The three-dimensional computer aided design (3D-CAD) software Solidworks is applied. The designed vertical winnower is able to separate different raw materials by adjusting the air speed and has been put into practical production to separate the Chinese traditional medicine with high separating effect.
\end{abstract}

Key words: Winnowing technology; Vertical winnower; 3D-CAD

\section{INTRODUCTION}

The winnowing technology has been used in agriculture for a long time as winnowing method, to separate chaff and broken rice stalk from the rice after harvest and threshing. Along with development of the industries such as mining and metallurgy industry, many kinds of winnowers have sprung up and were used in the field of mining, agriculture, pharmaceutical industry and urban construction, to separate such raw materials as Chinese traditional medicine, soybean and hops. Owing to the higher demands of separation, the winnowing technology and the structure design of the winnowers receive

\footnotetext{
Please use the following format when citing this chapter:
}

Bao, Y., Lin, S. and Weng, L., 2009, in IFIP International Federation for Information Processing, Volume 294, Computer and Computing Technologies in Agriculture II, Volume 2, eds. D. Li, Z. Chunjiang, (Boston: Springer), pp. 1497-1503. 
more and more attention (Ma Jiguang,2001; Wu Jianzhang et al, 2002; Shapiro et al, 2005).

In the developed countries such as Europe and American, the production of the gravitational winnower is $1 \sim 15 \mathrm{t} / \mathrm{h}$. The shape of the table board may be triangle, rectangle and mixed shape. The air may be negative pressure or positive pressure. Mary series of gravitational winnowers have been developed featuring excellent technique, stable performance, low noise and high reliability. Among these manufacturing factory, WESTRUP in Denmark, HEID in Austria, PETKUS in Germany, OLIVER, LMC and CRIPPEN in American are the more famous. But in China the users depend mostly on pure import or reforming the imported machine (Hu Zhichao,2002; Liu Xiang, 2004).

A vertical winnower is designed based on three-dimensional computer aided design, the shape, characteristic, processing condition and material quality of winnower will display in computer with real size and shape, convenient for the data exchange and share. The three-dimensional virtual product model can be operated in several ways that cannot realize for the entity model, such as slitting of the parts or assemblies, assembling and moving simulation in dimension interference. In the earlier period of designing, all the parts, tool equipments and assemblies are digital preassembled to reduce the design alteration, mistakes and reworks obviously (Tang Rongxi, 2005; Li Yan, 2007). The winnower was designed and developed successfully and applied in winnowing of Chinese traditional medicine with a good effect.

\section{THE PRINCIPLE OF WINNOWING}

Different materials have different movement under the action of vertical and horizontal (or gradient) air flow due to different aerodynamical properties, and may be separated accordingly. The aerodynamical properties include suspending velocity and flight coefficient, and so on. The suspending velocity is the ratio of the horizontal pressure and the gravity, while the flight coefficient is used to reflect the ability of the horizontal air flow to take away the materials.

The material with small suspending velocity can be blown farther under the horizontal air flow, and vice versa. Under the action of the vertical or gradient air flow, if the air flow velocity is larger than the suspending velocity, the material will be carried away. But if the air flow velocity is smaller than the suspending velocity, the material will go down. Therefore the heavy material is separated from the light material (Guo RenNing et al, 2005). 
When material is in the horizontal or gradient air-flow, considering the action of the gravity $G$ and air-flow pressure $P$, it will move along the direction of resultant force $\mathrm{T}$ and the trail is a parabola, as shown in Fig.1 (Li Shijiu, 1992).

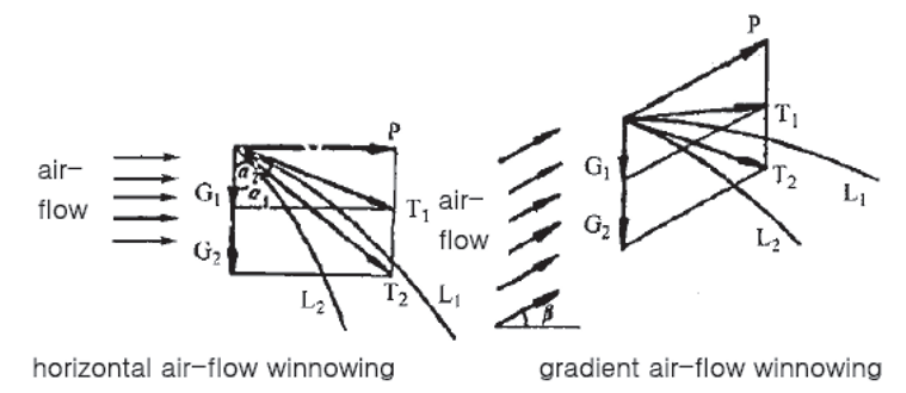

Fig. 1: The movement of material in the horizontal and gradient air-flow

In horizontal or gradient air-flow, light materials is blown farther than the heavy one, i.e. the bigger the angle between gravity $\mathrm{G}$ and resultant force $\mathrm{T}$ (the direction of $\mathrm{T}$ is close to the horizontal), the materials is blown farther, and Vice versa. Included angle $\alpha$ depends on following equation,

$$
\operatorname{tg} \alpha=\frac{P}{G}, \text { where } \operatorname{tg} \alpha \text { is the flight coefficient. }
$$

Different materials have different flight coefficient under the same horizontal or gradient air-flow. The larger gravity results to the smaller ${ }^{\operatorname{tg} \alpha}$ so that the material will be blown closely. Whereas the smaller gravity leads to the larger ${ }^{\operatorname{tg} \alpha}$ so that the will be blown far. So if only the suitable air flow velocity is chosen, the heavy and light materials can be separated accordingly.

In designing the vertical winnower, the gradient (about $30^{\circ}$ ) air flow is used to winnow and remove impurities, since it owns farther flying distance thereby has better separating effect.

\section{SETTLEMENT COMPUTATION AND AIR DUCT STRUCTURAL DESIGN}

\subsection{Settlement computation}

If the flow rate in the air duct equals to the settling velocity, particles in the air will be taken away, this velocity is called carryover velocity. It is 
apparent that the carryover velocity is equal to settling velocity for single particle (Li Shijiu, 1992).

The carryover velocity is: $U=\sqrt{\frac{4 g d_{p}\left(\rho_{s}-\rho_{f}\right)}{3 C_{p} \rho_{f}}}$.

For given particle and fluid, the gravity and buoyancy is determinate, the resistance changes with the falling velocity. At the beginning of the settlement, the falling velocity and resistance are zero. Then as the falling velocity increases, the resistance increases correspondingly balancing with the settling force, so that the acceleration became zero. Hereafter the particles fall down with uniform velocity called settling velocity, as shown in Fig. 2.

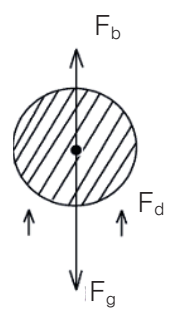

Fig.2: Forces balanced on a spherical particle

Considering a spherical particle with diameter $\mathrm{d}$, the density $\rho$ s, settling down in the air with density $\rho$, there are gravity $F_{g}$, buoyancy $F_{b}$ and resistance $F_{d}$ along the settling direction (vertical):

$$
F_{g}=\frac{\pi}{6} d^{3} \rho_{s} g \quad F_{b}=\frac{\pi}{6} d^{3} \rho g \quad F_{d}=\xi A \frac{\rho u^{2}}{2}=\frac{\xi \pi d^{2} \rho u^{2}}{8}
$$

Because the particle is in a balance, $F_{g}-F_{b}-F_{d}=0$

The flow can be divided into three regions: laminar flow, transition flow and turbulent flow, depending on the Reynolds number, as shown in Table 1,

$$
\mathrm{R}_{\mathrm{e} t}=\frac{d u_{t} \rho}{\mu}
$$

Table 1 Computation of gravitational settling velocity

\begin{tabular}{ccc}
\hline Flow regions & Reynolds number & Gravitational settling velocity \\
\hline laminar flow & $\operatorname{Re}<2$ & $u_{t}=\frac{d^{2}(\rho s-\rho) g}{18 \mu}$ \\
transition flow & $2<\operatorname{Re}<500$ & $u_{t}=\sqrt[3]{\frac{4 g^{2}\left(\rho_{s}-\rho\right)^{2}}{225 \mu \rho}} d_{p}$ \\
turbulent flow & $500<\operatorname{Re}<200000$ & $u_{t}=1.74 \sqrt{\frac{d(\rho s-\rho) g}{\rho}}$ \\
\hline
\end{tabular}


Supposed the average density of Chinese traditional medicine is about 300 $\mathrm{kg} / \mathrm{m}^{3}$ and its equivalent average diameter is about $3 \mathrm{~mm}$. Considering at the room temperature and atmospheric pressure, the density of the air $1.2 \mathrm{~kg} / \mathrm{m}^{3}$, the reasonable air flow velocity can be computed to be $4.71 \mathrm{~m} / \mathrm{s}$ by trial-anderror method.

\subsection{Structural design of the air duct}

The core structure of a vertical winnower is the air duct, the duct design will influence the efficiency and precision of winnowing .According to the air flow velocity, the corresponding dimensions can be computed, and then structure of the air duct is designed with 3D software- solidworks.

The vertical winnower has a vertical section and a horizontal winnowing trunk. The raw materials enter into the vertical duct and then the winnowing trunk with the blown air, as shown in Fig.3. The air is blown into the winnower at inlet 1 . The raw materials are feed in 2 and then move toward the winnowing trunk. In this process, the heavy impurities such as small iron block and stone fall down from outlet 5. While the rest move into the winnowing trunk together with the air flow. The air flow rate and correspondingly the pressure must be adjustable to let most required materials come out from the outlet 4 . The light materials (like hair, cotton yarn, paper scraps) will come out of 3 . To ensure the airproof and good circulation, the flanges are welded to the air-inlet 1 and outlet 3 .

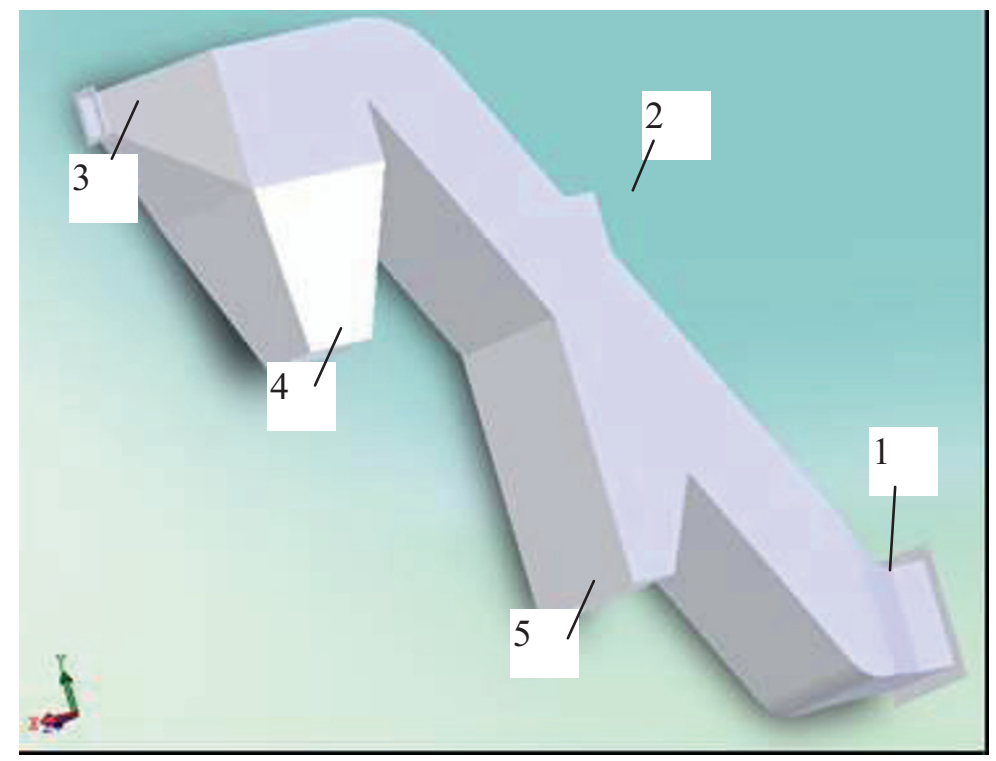

Fig.3: Air duct of the vertical winnower

1. air-inlet 2. raw materials inlet 3 .air outlet 4.product outlet 5 .heavy impurities outlet 
Considering the designed vertical winnower is mainly applied to separate medicine, seed etc, therefore all the materials used are stainless steel $1 \mathrm{Cr} 18 \mathrm{Ni9}$.

\subsection{Overall structure of winnower}

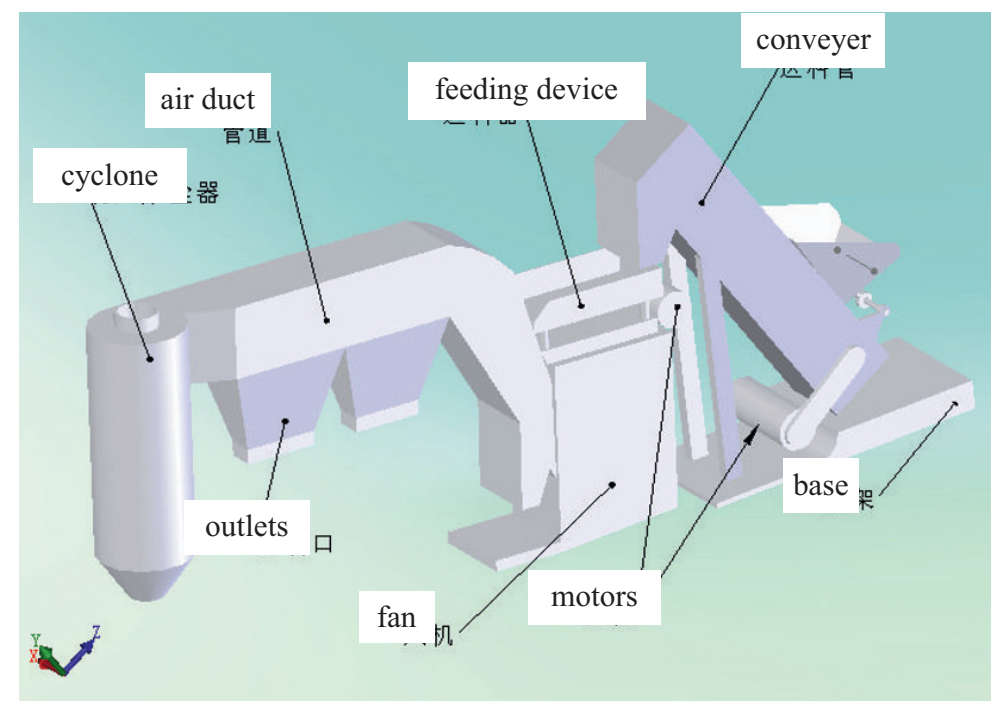

Fig.4: Working principle of the vertical winnower

As shown in Fig.4, The raw materials is conveyed to the feeding device by the conveyer and then fed into the air duct. Moving with the uniform airflow, the heavy materials (like iron blocks, stone and so on) and the products fall down from the outlets, while the light impurities (like hair, paper scraps) are blown into the cyclone to avoid environment polluted.

It is the most important in operating the vertical winnower to control the wind rate and the conveying velocity, depending on the granularity and density of the raw materials.

\section{CONCLUSIONS}

A vertical winnower is designed with $3 \mathrm{D}-\mathrm{CAD}$ software Solidworks and is able to separate the raw materials with different density by adjusting the air flow rate. The designed winnower has been put into some factories to separate the Chinese traditional medicine with good effect. The winnower is easy to maintenance, stable in operation and has healthful process.

The designed vertical winnower can also be applied to winnow other raw materials such as seed and soybean with a little modification and adjustment. 


\section{ACKNOWLEDGEMENTS}

This research was supported by Hangzhou Chunjiang Institute of Pharmacy machinery.

\section{REFERENCES}

Guo RenNing, Gao Chunyu, Ji Junhong. Numerical Simulation and Analysis to Inner Flow Field of Horizontal Air Flow Winnower. Mining \& Processing Equipment, 2005, 33(10): (57-59)

Hu Zhichao, Gu Renhong. On the nationalization of the winnowing equipment for the seeds. China Agriculture Mechanization, 2002,(3).

Li Shijiu, Zhou Xiaojun. pneumatic conveying theory and application. Beijing: China machine Press, 1992.

Li Yan. Three-Dimensional Structure Design for Ships by CADDS. Chinese Journal of Ship Research, 2007, 2 ( 1): 14-18

Liu Xiang. Structure and working principle of air fiber sifter. China forest products industry. 2004,31(6):30-31,45

M. Shapiro, V. Galperin. Air classification of solid particles: a review. Chemical Engineering and Processing 44 (2005): 279-285

Ma Jiguang. Development trend of the gravitational winnowers abroad. World agriculture, 2001,(7): 32-33.

Tang Rongxi. Popularization and rapid rise of 3D SolidWorks. Manufacture information engineering of China, 2005, 4:54

Wu Jianzhang, Zhu Yongyi. Study on Cereal Winnowing by Gas - Solid Fluidization. Cereal $\&$ feed industry, 2002, 6: 11-13 